\title{
Utilization Management Opportunities for Palivizumab for Prophylaxis of Respiratory Syncytial Virus Complications in Infants
}

\author{
Frederic R. Curtiss, PhD, RPh, CEBS, and Kathleen A. Fairman, MA
}

A survey conducted in December 2008 of 69 health plans representing over 83 million commercial, Medicare, and managed Medicaid members found that $81 \%$ of the health plans required prior authorization (PA) for coverage of palivizumab (Synagis). ${ }^{1}$ Palivizumab is commonly covered under the medical benefit (73\%) rather than the pharmacy benefit, and is ranked among the therapeutic areas with the highest priority for utilization management (UM). Health plans cite as the primary motivation for targeting palivizumab for UM intervention "to prevent inappropriate use," the same motivation attributed to UM interventions for growth hormones, blood cell stimulants, and omalizumab for asthma. ${ }^{1}$

Despite the importance of UM interventions and the prevalence of PA programs for the use of palivizumab in prophylaxis of respiratory syncytial virus (RSV), to date there have been no peer-reviewed analyses of the clinical, service, or cost outcomes of PA interventions for this high-cost injectable. In this issue of $J M C P$, Buckley et al. provide the first information in the medical literature regarding the outcomes of a PA intervention to determine coverage for palivizumab. Based on American Academy of Pediatrics (AAP) guidelines, this PA intervention was associated with similar or better clinical outcomes while saving about $\$ 2.4$ million in palivizumab drug cost over 3 RSV seasons from 2005 through 2008 in a health plan of about 500,000 members. ${ }^{2}$ Also in this issue of JMCP, Diehl et al. report the results of an exploratory study of the association between compliance with palivizumab and respiratory-related medical utilization in a small sample of children aged 0 to 24 months who met similar AAP-based PA criteria and were immunoprophylaxed during the 2006-2007 RSV season, finding no significant relationships between palivizumab compliance and RSV-related utilization. ${ }^{3}$

\section{Indications and Recommendations for Appropriate Use of Palivizumab}

Palivizumab was approved by the U.S. Food and Drug Administration (FDA) in June 1998 for "prophylaxis of serious lower respiratory tract disease, caused by respiratory syncytial virus, in pediatric patients at high risk of RSV disease.".4 The initial product label included the indication "for the prevention of serious lower respiratory tract disease caused by respiratory syncytial virus (RSV) in pediatric patients at high risk of RSV disease. Safety and efficacy were established in infants with bronchopulmonary dysplasia (BPD) and infants with a history of prematurity (=35 weeks gestational age)." ${ }^{5}$
AAP guidelines initially (1998) recommended that the appropriate use of palivizumab should be based on the age of the infant at the start of the RSV season: (a) children less than 2 years old with chronic lung disease (CLD) who required medical therapy within the 6 months prior to the RSV season, (b) infants less than 1 year old born up to 28 weeks gestation, and (c) infants less than 6 months old born between 29 and 32 weeks gestation. ${ }^{6}$ For infants with a gestational age of 32 to 35 weeks, the AAP guidelines suggested that palivizumab "should be reserved for those infants with additional risk factors" that "predispose to respiratory complications," including neurologic disease, young siblings, child care attendance, "exposure to tobacco smoke in the home, anticipated cardiac surgery, and distance to and availability of hospital care for severe respiratory illness." ${ }^{\prime \prime}$ The initial AAP guidelines in 1998 further suggested that many infants should not receive palivizumab despite "qualifying for the approved indications" because the "risk of rehospitalization for serious respiratory illness will be low, and the cost and logistical difficulties associated with prophylaxis may outweigh the potential benefits."6

The efficacy of palivizumab in prophylaxis of RSV-related hospitalization has been studied in 2 randomized controlled trials (RCTs). Palivizumab was initially approved by the FDA based on the IMpact-RSV trial (1998), which was conducted during a single RSV season (1996-1997) and randomized 1,502 children who at the start of the RSV season were either (a) aged 24 months or younger with bronchopulmonary dysplasia (BPD, now known as CLD) or (b) aged 6 months or younger with a history of premature birth (defined as 35 weeks gestation or less) to placebo or 5 monthly intramuscular (IM) injections of palivizumab 15 milligrams $(\mathrm{mg})$ per kilogram $(\mathrm{kg})$ administered over 150 days. In the IMpact-RSV trial, the incidence of RSV-related hospitalization was $10.6 \%(n=53)$ among 500 patients in the placebo group compared with $4.8 \%(n=48)$ among 1,002 patients in the palivizumab group $(P<0.001)$. The numbers needed to treat (NNT) in IMpact-RSV were 17.2 for the sample overall, ${ }^{8} 20.4$ for children with BPD (12.8\% placebo vs. $7.9 \%$ palivizumab, $P=0.038$ ), and 15.9 for premature infants without BPD (8.1\% placebo vs. $1.8 \%$ palivizumab, $P<0.001)$.

Patients with uncorrected congenital heart disease (CHD) were excluded from the IMpact-RSV trial. There was no mention of CHD in the initial palivizumab product label, and the AAP recommendations in 1998 indicated that palivizumab was "not recommended for children with cyanotic congenital 
heart disease." ${ }^{\prime \prime}$ The palivizumab product label was revised in July 2004 to include the additional indication for "children with hemodynamically significant CHD," based on the results of 1 clinical trial conducted over 4 consecutive RSV seasons among infants aged 2 years old or younger with hemodynamically significant CHD. ${ }^{9}$ This second RCT of palivizumab, conducted by Feltes et al. (2003), found a RSV-related hospitalization rate of $9.7 \%$ in the placebo group (63 of 648 patients) compared with 5.3\% (34 of 639 patients) in the palivizumab group (IM $15 \mathrm{mg}$ per $\mathrm{kg}$ ) followed for 150 days after randomization. ${ }^{10}$ The NNT for the Feltes et al. study was 22.7. ${ }^{8}$ RSV-related hospitalization is the primary endpoint in clinical analysis of the effectiveness of palivizumab, because there is no evidence that palivizumab affects mortality associated with RSV infection. ${ }^{11}$

Notably, both clinical trials cited on the palivizumab product label involved dosing and regimen completion rates that are implausible in the real world. Almost all patients in both RCTs completed the studies, and 92\%-93\% completed all 5 injections, exactly 30 days apart. ${ }^{7,10}$

\section{What is the True Incidence of RSV-Related Hospitalizations and ER Visits?}

In an analysis of the U.S. National Hospital Discharge Survey (NHDS) data for the 17-year period from 1980 to 1996, Shay et al. found an estimated 1.65 million hospitalizations for bronchiolitis among children younger than 5 years and estimated that there were 51,240 to 81,985 annual bronchiolitis hospitalizations related to RSV infection among children younger than 1 year of age. ${ }^{12}$ Shay et al. noted that prior to their research only the Institute of Medicine (IOM, 1985) had estimated a national rate of RSV-related hospitalizations, about 55,000 per year for infants younger than 1 year and about 36,500 for children aged 1 to 4 years. However, the IOM estimates were based on assumptions that $0.5 \%$ of children younger than 5 years who were infected with RSV would require hospitalization, and that infants younger than 1 year would account for $60 \%$ of the RSV-related hospitalizations. ${ }^{12}$

While this analysis by Shay et al. is widely referenced in the research literature as a benchmark for the incidence of RSVrelated hospitalizations, these researchers reached their estimate of RSV-related hospitalizations by assuming, based on previously published viral epidemiology studies, that RSV was the etiologic agent in 50\% to $80 \%$ of November through April hospitalizations for bronchiolitis in the NHDS database for 1994 to 1996. This estimation method was necessary because there were no specific International Classification of Diseases, Ninth Revision, Clinical Modification (ICD-9-CM) codes for RSV-related disease, other than RSV pneumonia (ICD-9-CM 480.1), until October 1996. ${ }^{13}$ Using the NHDS database for the 2-year period from January 2000 through December 2001 to identify hospital stays with the specific ICD-9-CM codes for RSV (466.11 for RSV bronchiolitis, 480.1 for RSV pneumonia, and 079.6 for RSV infection), Holman et al. found 221,450 RSV hospitalizations for infants younger than 1 year of age, of which 196,009 (88.5\%) were specific to RSV bronchiolitis. ${ }^{13}$ Annualized, the resulting count of approximately 111,000 RSV hospitalizations in U.S. infants in 2000-2001 suggests that Shay et al.'s figures may have been underestimates.

During the 17-year period from 1980 to 1996 studied by Shay et al., 57\% of the estimated 1.65 million hospitalizations for bronchiolitis were accounted for by children younger than 6 months and $81 \%$ by children younger than 1 year. Among children younger than 1 year, annual bronchiolitis hospitalization rates increased more than 2-fold, from $1.29 \%$ in 1980 to $3.12 \%$ in 1996. ${ }^{12}$ If the portion of bronchiolitis hospitalizations attributed to RSV was $50 \%$ to $80 \%$ in $1996,1.56 \%$ to $2.50 \%$ of children younger than 1 year were hospitalized with RSV-related disease in 1996. Probably representing the combined effects of change in coding methodology and a continued pattern of increasing RSV hospitalization rates, Holman et al. found in 2000-2001 that the rates of RSV hospitalizations per 1,000 live births were approximately $30.3(3.0 \%)$ for males and 24.4 (2.4\%) for females, or 27.4 (2.7\%) overall (Table 1$){ }^{13}$

Hall et al. found in population-based surveillance in 3 U.S. counties (Nashville, Rochester and Cincinnati) absolute rates of RSV-related hospitalizations of $1.7 \%$ for infants $0-5$ months of age, $0.5 \%$ for infants $6-11$ months of age, $0.3 \%$ for children 12 to 23 months of age, $0.04 \%$ for children 24 to 59 months of age, and $0.3 \%$ overall for children 0 to 59 months of age, over 4 RSV seasons (2000-2004). ${ }^{14}$ RSV is the apparent cause of 1 every 334 hospitalizations and 1 of every 38 ER visits in children younger than 60 months of age. ${ }^{14}$

In this issue of JMCP, Buckley et al. found RSV-related hospitalization rates of 6.4\% (40 of 629) in infants who were approved by the PA process to receive palivizumab, compared with $4.0 \%$ (14 of 348) for infants who were denied PA requests for coverage of palivizumab $(P=0.055)$, for a combined rate of $5.5 \%$ (54 infants with hospitalizations divided by 977 infants). ${ }^{2}$ This rate is considerably higher than the hospitalization rates for bronchiolitis estimated by Shay et al. for infants younger than 1 year of age for 1980 (1.3\%) and 1996 (3.1\%). ${ }^{12}$ The discrepancy is probably partly attributable to both the upward trend in RSV-related hospitalization suggested by the analyses of NHDS data ${ }^{12,13}$ and to the likely higher risk of the infants in Buckley et al.'s sample, whose physicians requested PA for palivizumab, compared with the U.S. infant population as a whole. There is also considerable variation in rates of RSV-related hospitalization among regions of the country. For infants under 6 months of age, Hall et al. found rates of RSV-related hospitalization of $1.3 \%$ in Nashville, $1.4 \%$ in Rochester, and 3.4\% in Cincinnati..$^{14}$ Notably, the American Southwest, where Buckley et al's study was conducted, had one of the highest RSV hospitalization rates in the country in 20002001; Holman et al. found rates of 4.8\% among American Indians in the southwestern United States and 3.7\% among all infants in the West. ${ }^{13}$ 


\section{TABLE 1 Rates of RSV-Related Hospitalization}

Source-Study Authors and Population

IMpact-RSV: ${ }^{7}$ children either (a) aged 2 years or younger who required continuing medical therapy

for CLD or (b) history of premature birth ( $\leq 35$ weeks gestation) and aged $<6$ months at start of RSV season

Children with CLD

Premature infants without CLD

Feltes et al. (2003): 10 children aged 2 years or younger with hemodynamically significant CHD

Holman et al.:13 U.S. population $<1$ year of age (NHDS database)

RSV Season

1996-1997

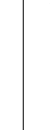

(196-1997

RSV-Related Hospitalization Rat

Placebo: $10.6 \%$

Palivizumab: $4.8 \%$

Placebo: $12.8 \%$

Palivizumab: $7.9 \%$

Placebo: $8.1 \%$

Palivizumab: $1.8 \%$

\begin{tabular}{c|l}
\hline 4 seasons: & Placebo: 9.7\% \\
1998-2002 & Palivizumab: 5.3\% \\
\hline
\end{tabular}

2 seasons: $\quad$ Male: $3.0 \%$

2000-2001 Female: 2.4\%

Age $<6$ mos.: $4.2 \%$

Age 6-11 mos.: 1.3\%

Overall: $2.7 \%$

Holman et al.:13 American Indians and Alaska Natives $<1$ year of age

2 seasons: $\quad$ Male: $3.7 \%$

2000-2001 Female: 3.2\%

Alaska: $7.1 \%$

Southwest: $4.8 \%$

Overall: $3.4 \%$

Hall et al.:14 3 U.S. counties (Nashville, Rochester, Cincinnati)

4 seasons:

2000-2004

Age 0-5 mos.: $1.7 \%$

Age 6-11 mos.: 0.5\%

Age 12-23 mos.: $0.3 \%$

Age 24-59 mos.: $0.04 \%$

Age $0-59$ mos.: $0.3 \%$

Frogel et al.:15 Palivizumab Outcomes Registry - U.S. population ${ }^{a}$

4 seasons: $\quad$ Male: $1.5 \%$

2000-2004 Female 1.0\%

Overall: $1.3 \%$

GA $<32$ wks.: $1.8 \%$

GA $32-35$ wks.: $0.8 \%$

GA > 35 wks.: $1.1 \%$

CLD: $2.4 \%$

CHD: $1.9 \%$

CAA/SND: $2.1 \%$

Medicaid: $1.6 \%$

2000-2001 RSV season: $2.9 \%$

2001-2002 RSV season: 1.5\%

2002-2003 RSV season: $1.1 \%$

2003-2004 RSV season: 0.7\%

Buckley et al.:2 MCO with PA for palivizumab

Diehl et al.: ${ }^{3}$ MCO with PA for palivizumaba

aAll subjects received at least 1 dose of palivizumab.

$C A A / S N D=$ congenital airway abnormality or severe neuromuscular disease; $C H D=$ congenital heart disease; $C L D=$ chronic lung disease; GA = gestational age;

$M C O=$ managed care organization; mos = months; NHDS=National Hospital Discharge Survey; $P A=$ prior authorization; RSV=respiratory syncytial virus; whs = weeks.

\section{How Much Does Palivizumab Reduce the Incidence of RSV-Related Hospitalization and ER Visits?}

This is the $\$ 64,000$ question, or more accurately, the $\$ 7,000$ per patient per RSV season question. Buckley et al. found that the real-world cost for prophylaxis of RSV in a health plan in 2005-2008 was about \$6,950 per infant per season in direct drug costs. ${ }^{2}$ Buckley et al. assessed health plan cost plus member cost (i.e., managed care organization [MCO] allowed charge for palivizumab), whereas Diehl et al. found health plan drug cost (after subtraction of member cost share) for palivizumab to average $\$ 8,142$ per infant in the 2006-2007 RSV season, or a mean of
$\$ 9,308$ for 73 compliant infants versus $\$ 7,647$ for 172 noncompliant infants.

In addition to the aforementioned nonsignificant difference in RSV-related inpatient hospitalizations between PA-approved and PA-denied infants, Buckley et al. found a higher rate of RSVrelated ER visits in the PA-approved group $(n=14,2.2 \%) \mathrm{com}$ pared with the PA-denied group $(n=5,1.4 \% ; P=0.019)$, and $6.6 \%$ of the infants in the PA-approved group had either a RSV-related hospitalization or RSV-related ER visit compared with $4.3 \%$ of the infants in the group denied PA for coverage of palivizumab $(P=0.060)$. 
The rate of RSV-related hospitalization varies greatly among subgroups of infants with certain risk factors. As noted above, the initial product label for palivizumab did not specify CHD as an indication for palivizumab. ${ }^{5}$ The label was revised in July 2004 to include the indication for "children with hemodynamically significant CHD," based on the RCT results reported by Feltes et al. for RSV seasons 1998-2002. ${ }^{10}$ In a separate analysis of data from the Palivizumab Outcomes Registry for 19,548 children who received at least 1 dose of palivizumab during 1 or more of 4 RSV seasons (2000-2004), 7.7\% ( $n=1,500)$ had a medical history of CHD. ${ }^{15}$ Frogel et al. found a 1.2\% hospitalization rate for infants without $\mathrm{CHD}$ versus a $1.9 \%$ hospitalization rate among 1,490 infants with CHD ( $P=0.03)$; within the CHD subgroup, the rate declined sharply from $4.3 \%$ in the 2000-2001 season and $2.8 \%$ in the $2001-2002$ season to $1.4 \%-1.5 \%$ in the 2 consecutive seasons from 2002-2004.15

Of the risk factors, the highest rates of RSV-related hospitalization for children in the Palivizumab Outcomes Registry from 2000 to 2004 were found for children with $\operatorname{CLD}(2.4 \%$ of 4,329), versus $2.1 \%$ of 1,122 children with congenital airway abnormality or severe neuromuscular disease, $1.8 \%$ of 7,786 children with gestational age less than 32 weeks, $1.6 \%$ of 9,228 children with Medicaid coverage, $1.6 \%$ of 7,260 children born into families with 3 or more children, and 1.5\% for 10,481 males. ${ }^{15}$ Univariate logistic regression analysis showed that gestational age of 32-35 weeks was not a predictor of RSV-related hospitalization, with an absolute rate of $0.8 \%$ compared with $1.1 \%$ for gestational age greater than 35 weeks $(P=0.16)$, but there was a higher rate of RSV-related hospitalization for single births (1.4\%) versus multiple births $(1.0 \%, P=0.03)$.

In the study by Hall et al. of 5,067 children less than 5 years of age who were either hospitalized from 2000-2004 or seen as outpatients from 2002-2004 for acute respiratory infection in 3 U.S. counties during the RSV season, 18\% $(n=919)$ had RSV infections. ${ }^{14}$ The RSV infection rate was 20\% in hospital inpatients, $18 \%$ in the ER, and $15 \%$ in office visits. Palivizumab was found to have been administered to 174 of 1,765 (9.9\%) highrisk children (defined as chronic pulmonary, cardiac, kidney or immunodeficiency disease; cancer; or sickle cell anemia); and to 140 of 657 (21.3\%) of premature infants (gestational age less than 36 weeks). Particularly important to the subject of the effectiveness of palivizumab in real-world practice, $9.8 \%(n=17)$ of 174 high-risk patients who had received palivizumab and $11.4 \%$ $(\mathrm{n}=16)$ of 140 premature births who had received palivizumab were hospitalized with RSV infection..$^{14}$

\section{Does Compliance with the Monthly Regimen for Palivizumab Matter?}

In this issue of JMCP, Diehl et al. found in real-world analysis of palivizumab utilization in an MCO with approximately 400,000 Medicaid and commercially insured members that RSV-related hospitalizations occurred in none of 73 infants determined to be compliant with palivizumab versus 2 of 172 (1.2\%) noncompliant infants $(P=1.00){ }^{3}$ Diehl et al. defined compliance as meeting all 3 of the following criteria: (a) fill date for the first palivizumab pharmacy claim within 37 days of the newborn hospitalization discharge date; (b) fill dates for all subsequent palivizumab pharmacy claims within 37 days of the previous fill date; and (c) up to 6 palivizumab pharmacy claims for babies born prior to the RSV season, or sequential claims from birth through the end of the season for babies born during the season.

Finding comparative data for the results reported by Diehl et al. is not straightforward. When compliance is defined as receipt of all palivizumab doses within a mean of 35 days of the previous dose, Frogel et al. in this issue of JMCP cite RSV-related hospitalization rates of $1.4 \%$ for children compliant with the palivizumab regimen compared with $3.1 \%$ in children determined to be noncompliant $(P<0.001) .{ }^{16}$ However, these are unpublished data that were collected via telephone survey and reported only in a poster abstract presented by Berger et al. in 2003, ${ }^{17}$ with no control for confounding variables. Using data derived from the Palivizumab Outcomes Registry, Frogel et al. found no association between RSV-related hospitalizations and compliance defined as the number of expected palivizumab injections. However, by the alternate measure of compliance defined as receipt of each dose within 35 days of the previous dose among patients with at least 2 doses, the rate of RSV-related hospitalization was $1.7 \%$ for noncompliant children versus $1.2 \%$ for compliant children $(P=0.007) .{ }^{15}$

Although this latter definition comes close to that used by Diehl et al., critically important methodological differences make comparing the results of the studies problematic. First, the Palivizumab Outcomes Registry Study measured compliance beginning with the month that the first injection was administered. In contrast, Diehl et al. defined infants whose injections did not begin within 37 days of the initial newborn discharge as noncompliant. Thus, an infant whose physician chose to initiate palivizumab immunoprophylaxis more than 37 days after birth but who maintained regular injections after that point was defined as compliant by the Palivizumab Outcomes Registry Study authors but noncompliant by Diehl et al. Second, RSV hospitalizations were measured using virology testing in the Palivizumab Outcomes Registry Study but using diagnoses recorded in claims data in the Diehl et al. study. Third, as often happens in real-world analyses of MCO outcomes, the Diehl et al. study was underpowered. Diehl et al.'s cohort sizes of 73 compliant and 172 noncompliant infants fall far short of the 310 cases in each group that would have been necessary for $80 \%$ power (2-tailed test, alpha 0.05) to detect a difference in RSV hospitalization rates of $11 \%$ versus $5 \%$, the results obtained in the IMpact-RSV trial for placebo- and palivizumab-treated infants, respectively. ${ }^{7}$ 


\section{Does Administration of Palivizumab by a Home Health Nurse Improve Clinical Outcomes?}

A primary message in the subject review by Frogel et al. in this issue of JMCP has to do with the potential value of palivizumab administered by home health care as "a key component in ensuring compliance during the RSV season."16 Frogel et al. summarize what is known about RSV-related hospitalizations and other outcomes associated with compliance with the palivizumab oncemonthly regimen based on 3 peer-reviewed studies and 7 poster abstracts that compared administration of the drug in the clinic or office versus home health care. Not surprisingly, in most studies the home care method of administration was associated with increased compliance with palivizumab immunoprophylaxis. ${ }^{16}$

However, whether improved clinical outcomes might accrue from home health administration of palivizumab is doubtful based on currently available evidence. Of the 10 studies of home health administration included in Frogel et al.'s review, 6 compared hospitalization rates for palivizumab administration by home health versus clinic or office settings. Of those, only 2 found significant differences between home health and clinic/ office administration, 1 of which assessed all-cause hospitalizations. Of the 5 studies to assess the percentage of babies with a hospitalization attributable to RSV, only 1, the Palivizumab Outcomes Registry Study, found significantly lower RSV hospitalization rates in home health care as compared with clinic/office ( $0.4 \%$ versus $1.2 \%$ for the sample overall, $P=0.014 ; 0.6 \%$ vs. $1.6 \%$ for a subgroup of Medicaid-enrolled infants, $P=0.02){ }^{15}$

Thus, absent consistent evidence of clinical benefit, Frogel et al. base their advocacy of home health administration in part on a theory that "a home-based delivery system might offer some additional benefit of decreasing exposure of the infant to pathogens, including RSV, in the clinic or office setting." ${ }^{\prime \prime 6}$ Frogel et al. proffer support for this assertion by citing Hand et al.'s brief report of a study that did not measure exposure to pathogens but in which the authors wrote that they "speculate that the decreased number of medical visits and hospitalizations may be a function of decreased exposure of the high-risk infant to the clinic environment."18 This is a curious citation by Frogel et al. because they acknowledge earlier in their review article that the report by Hand et al. suffers from the shortcoming that the research did not assess whether the hospitalizations reported during the RSV season (defined as October 1, through April 30) were actually attributable to RSV. ${ }^{16,18}$

Actually, there are a number of shortcomings in the report by Hand et al., which bring into question the "evidence" produced from research on the subject of clinic/office versus home health nurse administration of palivizumab. First, Hand et al. compared RSV hospitalization rates across 2 RSV seasons, clinic-based administration for the 2000-2001 RSV season versus homebased administration in the 2001-2002 RSV season. Second, the authors do not report important methodological details, such as the number of patients lost to follow-up. Third, the authors mention a subgroup analysis of patients 100\% compliant with palivizumab but present no data or counts to support the claim that hospitalizations were lower in the home-based arm, and the number of patients cited $(n=155)$ does not match the count of 171 patients $100 \%$ compliant across the 2 RSV seasons that is shown in the sole data table in their brief report. Fourth, the study by Hand et al. is not generalizable because all infants were Medicaid eligible (and Medicaid covered the service for home administration of palivizumab). Fifth, the cost of home-based services was not addressed; and sixth, there is no disclosure of funding or financial conflicts of interest in this brief report published in 2008 from data collected 6-7 years earlier. In short, the study by Hand et al. fails the test of quality of evidence, ${ }^{19}$ and the mean per patient number of hospitalizations reported by Hand et al., 1.01 in the clinic-based group versus 0.35 in the home-based group, are all-cause, not documented as RSV-related.

At a more basic level for MCOs, the practical value of using home health care to prevent exposure to pathogens in physician offices is markedly unclear in a patient population targeted to receive therapy in part because of exposure to pathogens in child care, an AAP risk criterion for palivizumab use. ${ }^{6,20,21}$ Notably, the proportion of Palivizumab Outcomes Registry Study patients who were either enrolled in child care themselves or were living with an enrolled sibling more than doubled from $22.5 \%$ in 2000 2001 to $51.4 \%$ in $2003-2004 .{ }^{15}$

\section{AAP Guidance in 2009 Narrowed the Scope of Appropriate Use of Palivizumab}

In September 2009, the Committee on Infectious Diseases of the AAP modified its 2003 and 2006 clinical practice guidelines and 2006 Red Book recommendations for the use of palivizumab for the prevention of RSV infections. The 2009 AAP policy statement narrowed significantly the target population for receipt of palivizumab prophylaxis, particularly for infants with a gestational age of 32 weeks to 35 weeks (Table 2) ${ }^{20,21}$ By far the most dramatic change is limitation of palivizumab to the lesser of 3 doses or 90 days of age, in premature infants born between 32 weeks 0 days and 34 weeks 6 days who do not have CHD, CLD, neuromuscular disease, or congenital abnormalities of the airways. Previously, a maximum of 5 doses was recommended for all high-risk infants. Second, the gestational age eligible for recommended prophylaxis was reduced by 1 day, from 35 weeks 0 days in the 2006 Red Book to 34 weeks 6 days in the AAP 2009 policy statement; this change means that the AAP 2009 policy statement deviates slightly from the FDA-approved labeling that defines prematurity as gestational age 35 weeks or less. ${ }^{22}$

The third important change that effectively reduces the target population is reduction in the scope and definition of risk factors that qualify for palivizumab prophylaxis in the subgroup of infants born from 32 weeks 0 days through 34 weeks 6 days gestational age. In addition to gestational age and age younger than 6 months at the start of the RSV season, the 2006 Red Book 


\section{TABLE 2 Key Eligibility Criteria for Palivizumab in the AAP Recommendations in 2006 and 2009}

\begin{tabular}{|c|c|c|}
\hline Category of Use Criteria & AAP 2006 Recommendations $^{a}$ & AAP 2009 Recommendations ${ }^{b}$ \\
\hline Gestational age & - $\leq 35$ weeks & $\begin{array}{l}\text { - } 32 \text { weeks } 0 \text { days through } 34 \text { weeks } 6 \text { days (i.e., excludes } 35 \\
\text { week } 0 \text { day infants) }\end{array}$ \\
\hline $\begin{array}{l}\text { Maximum } 5 \text { doses per } \\
\text { RSV season }\end{array}$ & $\begin{array}{l}\text { - "Palivizumab prophylaxis should be considered for infants } \\
\text { and children younger than } 24 \text { months of age with CLD of } \\
\text { prematurity who have required medical therapyc for CLD } \\
\text { within } 6 \text { months before the start of the RSV season. Patients } \\
\text { with more severe CLD who continue to require medical } \\
\text { therapy may benefit from prophylaxis during a second } \\
\text { RSV season. Data are limited regarding the effectiveness of } \\
\text { palivizumab during the second year of life." }\end{array}$ & $\begin{array}{l}\text { - "Palivizumab prophylaxis may be considered for infants and } \\
\text { children younger than } 24 \text { months with CLD who receive } \\
\text { medical therapyc for CLD within } 6 \text { months before the start } \\
\text { of the RSV season... Patients with the most severe CLD } \\
\text { who continue to require medical therapy may benefit from } \\
\text { prophylaxis during a second RSV season. Data are limited } \\
\text { regarding the effectiveness of palivizumab during the second } \\
\text { year of life." }\end{array}$ \\
\hline
\end{tabular}

- <24 months of age with hemodynamically significant cyanotic and acyanotic $\mathrm{CHD}^{\mathrm{d}}$ and require medical therapy; base decision on prophylaxis on "degree of physiologic cardiovascular compromise." Benefit of prophylaxis is most likely in infants who (a) are receiving medication to control CHF, (b) have moderate to severe pulmonary hypertension, or (c) have cyanotic heart disease.

- Premature infants with gestational age 29 weeks to 32 weeks with or without CLD and $<6$ months of age at the start of their first RSV season

- $<24$ months of age with hemodynamically significant cyanotic and acyanotic $\mathrm{CHD}^{\mathrm{d}}$ and require medical therapy; base decision on prophylaxis on "degree of physiologic cardiovascular compromise." Benefit of prophylaxis is most likely in infants who (a) are receiving medication to control CHF, (b) have moderate to severe pulmonary hypertension, or (c) have cyanotic heart disease.

- Premature infants born on before 32 weeks (up to 31 weeks 6 days) gestation with or without CLD "may benefit most from prophylaxis up to 6 months of age."

- Premature infants born at 28 weeks gestation or earlier with or without CLD and $<12$ months of age at the start of their first RSV season

- Premature infants born at 28 weeks gestation or earlier with or without CLD and $<12$ months of age at the start of the RSV season

- For infants born between 32 weeks, 1 day and 35 weeks, 0 days gestation, "prophylaxis should be reserved for infants... who are at greatest risk of severe infection and who are younger than 6 months of age at the start of the RSV season." $f$

Maximum 3 doses per

RSV season

Prematurity (gestational age 32-35 weeks) with risk factors
- Prematurity (32 weeks 1 day to 35 weeks 0 days gestational age) without CLD and $<6$ months of age at the start of the RSV season and 2 or more of 5 risk factors:

$>$ Child care attendance

> School-aged siblings

> Exposure to environmental air pollutants

$>$ Congenital abnormalities of the airways

$>$ Severe neuromuscular disease
- "Immunoprophylaxis may be considered for infants" up to 12 months of age who have either "significant congenital abnormalities of the airway or a neuromuscular condition that compromises handling of respiratory tract secretions."

- Premature infants with a gestational age of 32 weeks 0 days to 34 weeks 6 days with at least 1 risk factor and born during the RSV season or not older than 90 days at the start of the RSV season

- Prematurity (32 weeks 0 days through 34 weeks 6 days) and born 3 months before or during RSV season and at least 1 of 2 risk factors:

$>$ Child care attendance

$>1$ or more siblings or other children younger than 5 years living permanently in the same household

\footnotetext{
american Academy of Pediatrics 2006 clinical practice guideline on the diagnosis and management of bronchiolitis. ${ }^{20}$

${ }^{b}$ American Academy of Pediatrics 2009 policy statement on modified recommendations for the use of palivizumab for prevention of RSV infections. 21

'Medical therapy is defined as supplemental oxygen, bronchodilator, diuretic, or chronic corticosteroid therapy.

dHemodynamically significant CHD is defined in the AAP 2006 guideline as: "children with congenital heart disease who are receiving medication to control congestive heart failure, have moderate to severe pulmonary hypertension, or have cyanotic heart disease." 20 The AAP 2009 guideline does not define "hemodynamically significant," but defines the following heart disease conditions as "hemodynamically insignificant:" secundum atrial septal defect, small ventricular septal defect, pulmonic stenosis, uncomplicated aortic stenosis, mild coarctation of the aorta, and patent ductus arteriosus.

'The AAP definition of gestational age of 32 to 35 weeks was previously 32 weeks 1 day through 35 weeks 0 days. The 2009 recommendations redefined 32 to 35 weeks' gestation as 32 weeks 0 days through 34 weeks 6 days.

fThe AAP 2006 guidelines recommended consideration of prophylaxis for this group only if at least 2 of the following risk factors were present: child-care attendance, school-aged siblings, exposure to environmental air pollutants, congenital abnormalities of the airways, or severe neuromuscular disease. $A A P=$ American Academy of Pediatrics; $C H D=$ congenital heart disease; $C H F=$ congestive heart failure; $C L D=$ chronic lung disease; $R S V=$ respiratory syncytial virus.
}

suggested that at least 2 of 5 risk factors ("child care attendance, school-aged siblings, exposure to environmental air pollutants, congenital abnormalities of the airways, or severe neuromuscular disease") be present before considering the administration of palivizumab. ${ }^{20}$ Although the 2006 guidelines recognized that passive exposure to household smoke should not normally be considered as a risk factor, exposure to environmental air pollutants, which was included in the 2006 guidance, has been removed altogether as a risk factor from the 2009 guidance..$^{20,21}$ Now, 2 risk factors are recognized for consideration of the use of 
palivizumab in infants born between 32 weeks 0 days and 34 weeks 6 days and less than 90 days of age: (a) "infant attends child care;" or (b) "1 or more siblings or other children younger than 5 years live permanently in the child's household."21

Therefore, only 1 of the 5 former risk factors (day care attendance) survives into the 2009 policy statement in its previous form, and having school-aged siblings has been replaced by living with siblings or other children less than 5 years of age. The 2009 AAP policy statement attributes this change to "inconsistencies among studies that attempted to define risk factors identifying children at greatest risk of serious RSV lower respiratory tract disease" and an intent to better target the children at "highest risk of severe disease" by focusing on the 2 factors that most consistently predict risk of developing serious RSV lower respiratory tract disease. ${ }^{21}$

The 2009 AAP policy statement for the use of palivizumab to prevent RSV infections remains unchanged for infants with hemodynamically significant CHD, birth before 32 weeks 0 days, and CLD associated with prematurity. For these 3 groups, a maximum of 5 doses of palivizumab is still recommended. But, the 2009 AAP policy statement is also clear that when palivizumab prophylaxis is initiated after the start of the RSV season in these infants and children, all 5 doses will not be necessary. Table 1 in the 2009 policy statement also specifies the earliest start date for initiation of a maximum 5 monthly doses in infants or young children with CLD or CHD as July 1 in southeast Florida, September 15 in north-central and southwest Florida, and November 1 in "most other areas of the United States."21

One does not have to make a careful reading of the 2009 AAP policy statement on the use of palivizumab for the purpose of reducing RSV-related hospitalization to determine that the Committee on Infectious Diseases was (a) attentive to the high cost of the drug and the inconsistency in the medical literature regarding the risk factors that truly predict RSV-related hospitalization, and (b) more definitive than in previous guidelines in specifying groups of infants who will most likely not benefit from immunoprophylaxis. The 2009 AAP policy statement is clear that premature infants with gestational age from 32 weeks 0 days to 34 weeks 6 days are at higher risk of RSV-related hospitalization if born during the RSV season or if they are younger than 3 months of age at the start of the RSV season..$^{21}$ Among the groups of infants who should not receive palivizumab because of insufficiently increased risk of RSV or lack of evidence of benefit are the following: (a) infants with hemodynamically insignificant heart disease; (b) infants with lesions that can corrected by surgery, "unless they continue to require medication for congestive heart failure;" (c) "infants with mild cardiomyopathy who are not receiving medical therapy for the condition;" (d) immunocompromised children (except those with severe immunodeficiency or advanced acquired immune deficiency syndrome); and (e) patients with cystic fibrosis. ${ }^{21}$

\section{How Much is a PA Program for Palivizumab Really Worth?}

Questions about the relative value of treatments are unimportant in an environment of unlimited resources. However, managed care interventions assume that resources are not unlimited, and Buckley et al. found palivizumab drug cost savings of about $\$ 2.4$ million over 3 RSV seasons (2005-2008), without adverse clinical outcomes as measured by RSV-related ER visits and RSV-related hospitalizations. While the direct administrative costs for operation of this program are not reported, it is reasonable to estimate that a clinical pharmacist with convenient access to electronic medical records in an integrated health system might require 15 minutes on average to review each PA request to make a coverage determination based on explicit criteria. Therefore, the 1,090 PA requests processed by the health plan studied by Buckley et al. would require 272.5 hours or total administrative payroll costs of about $\$ 20,450$ over 3 RSV seasons to save $\$ 2.4$ million, assuming a 2,000 hour work-year and annual salary and fringe benefit costs of $\$ 150,000$. Not a bad return on investment, $\$ 100$ savings for $\$ 0.80$ administrative cost.

From another perspective, the PA program for palivizumab described by Buckley et al. saved their health plan of 500,000 members about $\$ 0.13$ per member per month (PMPM). Annual drug cost savings of $\$ 800,000$ may not seem so large when expressed as PMPM costs. On the other hand, if these PMPM savings are applied to the entire U.S. population of approximately 300 million people, the research by Buckley et al. suggests that there is nearly $\$ 500$ million in potentially avoidable palivizumab drug costs each year in the United States, some of which may be realized in the operation of existing PA programs. Palivizumab might be the perfect poster child for PA interventions in managed care that are evidenced-based and result in quality improvement by delivering equivalent or better clinical outcomes at lower cost.

\section{Authors}

FREDERIC R. CURTISS, PhD, RPh, CEBS, is Editor-in-Chief, and KATHLEEN A. FAIRMAN, MA, is Associate Editor and Senior Methodology Reviewer of the Journal of Managed Care Pharmacy.

AUTHOR CORRESPONDENCE: Frederic R. Curtiss, PhD, RPh, CEBS, Academy of Managed Care Pharmacy, 100 North Pitt St., Suite 400, Alexandria, VA 22314. Tel.: 830.935.4319;

E-mail:fcurtiss@amcp.org.

\section{DISCLOSURES}

The authors report no conflicts of interest related to the subjects or products discussed in this article. 


\section{REFERENCES}

1. EMD Serono. Serono Injectables Digest, Fifth edition. April 2009. Available at: https://olr.dwainc.com/2559Digest/PDF/EMDSerono5th EditionInjectablesDigest.pdf.

2. Buckley BC, Roylance D, Mitchell MP, Patel SM, Cannon HE, Dunn JD. Description of the outcomes of prior authorization of palivizumab for prevention of respiratory syncytial virus infection in a managed care organization. J Manag Care Pharm. 2010;16(1):15-22. Available at: http://www.amcp. org/data/jmcp/15-22.pdf.

3. Diehl JL, Daw JR, Coley KC, Rayburg R. Medical utilization associated with palivizumab compliance in a commercial and managed Medicaid health plan. J Manag Care Pharm. 2010;16(1):23-31. Available at: http://www. amcp.org/data/jmcp/23-31.pdf. Accessed December 30, 2009.

4. U.S. Food and Drug Administration. Product approval information, reference no. 97-1359, palivizumab. June 19, 1998. Available at: http://www. accessdata.fda.gov/drugsatfda_docs/appletter/1998/palimed061998L.htm. Accessed November 24, 2009.

5. U.S. Food and Drug Administration. Product label. Synagis (palivizumab) for intramuscular administration. December 2, 1999. Available at: http:// www.accessdata.fda.gov/drugsatfda_docs/label/2002/palimed102302LB.pdf. Accessed November 24, 2009.

6. American Academy of Pediatrics Committee on Infectious Diseases and Committee on Fetus and Newborn. Prevention of respiratory syncytial virus infections: indications for the use of palivizumab and update on the use of RSV-IGIV. Pediatrics. 1998;102(5):1211-16. Available at: http://pediatrics.aappublications.org/cgi/content/full/102/5/1211. Accessed December 18, 2009.

7. The IMpact-RSV Study Group. Palivizumab, a humanized respiratory syncytial virus monoclonal antibody, reduces hospitalization from respiratory syncytial virus infection in high-risk infants. Pediatrics. 1998;102(3):531-37.

8. Canadian Agency for Drugs and Technologies in Health. Palivizumab prophylaxis against respiratory syncytial virus. Issue 80, March 2007. Available at: http://www.cadth.ca/media/pdf/I3006_tr_Palivizumab_e.pdf. Accessed December 13, 2009.

9. U.S. Food and Drug Administration. Product label. Synagis (palivizumab) for intramuscular administration. July 24, 2004. Available at: http://www. accessdata.fda.gov/drugsatfda_docs/label/2004/103770_s5059_lbl.pdf. Accessed November 24, 2009

10. Feltes TF, Cabalka AK, Meissner HC, et al.; Cardiac Synagis Study Group. Palivizumab prophylaxis reduces hospitalization due to respiratory syncytial virus in young children with hemodynamically significant congenital heart disease. J Pediatr. 2003;143(4):532-40.

11. Meissner HC, Long SS. Respiratory syncytial virus infection and recurrent wheezing: a complex relationship. J Pediatr. 2007;15(1):6-7.
12. Shay DK, Holman RC, Newman RD, et al. Bronchiolitis-associated hospitalizations among US children, 1980-1996. JAMA. 1999;282(15):1440-46. Available at: http://jama.ama-assn.org/cgi/reprint/282/15/1440. Accessed December 6, 2009.

13. Holman RC, Curns AT, Cheek JE, et al. Respiratory syncytial virus hospitalizations among American Indian and Alaska native infants and the general United States infant population. Pediatrics. 2004;114:e437-e444. Available at: http://pediatrics.aappublications.org/cgi/reprint/114/4/e437. Accessed December 18, 2009.

14. Hall CB, Weinberg GA, Iwane MK, et al. The burden of respiratory syncytial virus infection in young children. N Engl J Med. 2009;360(6):588-98. Available at: http://content.nejm.org/cgi/reprint/360/6/588.pdf. Accessed December 12, 2009

15. Frogel M, Nerwen C, Cohen A, VanVeldhuisen P, Harrington M, Boron M; Palivizumab Outcomes Registry Group. Prevention of hospitalization due to respiratory syncytial virus: results from the Palivizumab Outcomes Registry. J Perinatol. 2008;28(7):511-17.

16. Frogel MP, Stewart DL, Hoopes M, Fernandes AW, Mahadevia PJ. A systematic review of compliance with palivizumab administration for RSV immunoprophylaxis. J Manag Care Pharm. 2010;16(1):46-58. Available at: http://www.amcp.org/data/jmcp/46-58.pdf.

17. Berger J, Fensterheim L, O'Rourke J, et al. The importance of Synagis compliance in preventing hospitalizations [abstract]. Pediatr Res. 2003;53 (4Pt 2):468A-9. Poster presented at: Pediatric Academic Societies' Annual Meeting; May 3-6, 2003; Seattle, WA.

18. Hand IL, Noble L, Geiss D, Shotkin A. Respiratory syncytial virus immunoprophylaxis in an urban population: a comparison of delivery strategies and outcomes. Ped Infect Dis J. 2008;27(2):174-75.

19. Vandenbroucke JP, von Elm E, Altman DG, et al. Strengthening the reporting of observational studies in epidemiology (STROBE): explanation and elaboration. Ann Intern Med. 2007;147(8):W-163-W-194. Available at: www.annals.org/cgi/reprint/147/8/W-163.pdf. Accessed December 12, 2009.

20. Subcommittee on Diagnosis and Management of Bronchiolitis. Clinical practice guideline. Diagnosis and management of bronchiolitis. Pediatrics. 2006;118(4):1774-93. Available at: http://pediatrics.aappublications.org/cgi/ reprint/118/4/1774. Accessed December 13, 2009.

21. Committee on Infectious Diseases. Policy statement-modified recommendations for use of palivizumab for prevention of respiratory syncytial virus infections. Pediatrics. 2009:124(6):1-8. Available at: http://pediatrics. aappublications.org/cgi/reprint/peds.2009-2345vl. Accessed December 13, 2009.

22. Synagis (palivizumab) for intramuscular injection. Prescribing information. MedImmune. March 2009. Available at: http://www.medimmune.com/ pdf/products/synagis_pi.pdf. Accessed December 13, 2009. 